\title{
Rehabilitación del intestino utilizando un análogo semisintético del péptido 2 similar al glucagón. Primera experiencia con teduglutide en Uruguay
}

Intestinal rehabilitation using semisynthetic glucagon-like peptide-2 analogue. First experience with teduglutide in Uruguay.

Reabilitação do intestino usando um análogo semi-sintético do peptídeo 2 semelhante ao glucagon. Primeira experiência com teduglutide no Uruguai

\section{Eduardo Moreira ${ }^{1}$, Lourdes Silva², Estela Olano ${ }^{3}$, Héctor Solar ${ }^{4}$}

\section{Resumen}

La falla intestinal crónica (FIC) o tipo III es una condición invalidante, y la nutrición parenteral crónica (NPC) domiciliaria es el tratamiento que permite a estos pacientes mantenerse con vida. Sin embargo, solamente uno de cada tres países latinoamericanos cuentan con ese recurso, y sus complicaciones no son infrecuentes. Estas complicaciones son las principales indicaciones para trasplante intestinal, un procedimiento que en la mayoría de los países de ingresos medios no se ha desarrollado y no ha presentado los resultados esperados. En los últimos años, la rehabilitación intestinal a nivel mundial ha mejorado sustancialmente con el uso de análogos semisintéticos del péptido 2 similares al glucagón, existiendo cada vez mayor evidencia que demuestra la posibilidad de rehabilitación intestinal e independencia de la NPC con este fármaco, incluso en pacientes con anatomía desfavorable. Estos resultados han permitido mejorar la supervivencia y la calidad de vida de pacientes con FIC y, en muchas ocasiones, prescindir del trasplante. El paciente del caso que presentamos es el primero en recibir esta terapéutica en nuestro país. En este artículo analizamos la respuesta precoz favorable al tratamiento y sus perspectivas a futuro.

Palabras clave: Falla intestinal crónica Síndrome del intestino corto Nutrición parenteral en el domicilio Péptido 2 similar al glucagón Teduglutide
Key words: $\quad$ Chronic intestinal failure Short bowel syndrome Parenteral nutrition, home Glucagon-like peptide 2 Teduglutide

\footnotetext{
1. Médico Intensivista. UCI del Hospital Maciel. Integrante del Equipo Multidisciplinario de Terapia Nutricional de la Unidad de Nutrición e Insuficiencia Intestinal del Hospital Maciel. ASSE. Montevideo, Uruguay.

2. Licenciada en Enfermería. Integrante del Equipo Multidisciplinario de Terapia Nutricional. De la Unidad de Nutrición e Insuficiencia Intestinal del Hospital Maciel. ASSE. Montevideo, Uruguay.

3. Médico Intensivista. Coordinadora del Equipo Multidisciplinario de Terapia Nutricional de la Unidad de Nutrición e Insuficiencia Intestinal del Hospital Maciel. ASSE. Montevideo, Uruguay. Jefe de la Unidad de Nutrición Especial del Sanatorio CASMU. Montevideo, Uruguay.

4. Médico Intensivista. Coordinador de la Unidad de Insuficiencia Intestinal, Rehabilitación y Trasplantes, Fundación Favaloro, Hospital Universitario, Buenos Aires, Argentina.

Equipo Multidisciplinario de Terapia Nutricional (EMTN). Unidad de Nutrición e Insuficiencia Intestinal (UNII) del Hospital Maciel. Falla Intestinal, Grupo Montevideo.

Los autores declaran no tener conflictos de intereses.

Se solicitó consentimiento informado al paciente para la publicación.

Correspondencia: Dr. Eduardo Moreira. Correo electrónico: dreduardo.moreira@gmail.com

Fecha de recibido: $21 / 2 / 2021$

Fecha de aprobado: 16/6/2021

Attribution-NonCommercial 4.0 International (CC BY-NC 4.0)
} 


\section{Introducción}

Los pacientes adultos con menos de $200 \mathrm{~cm}$ de intestino delgado se definen como pacientes con síndrome de intestino corto (SIC). Las enterectomías secundarias a la isquemia intestinal, las complicaciones quirúrgicas, incluida la cirugía bariátrica, las enterectomías masivas y la catástrofe abdominal por patología traumática se han convertido en las principales causas ${ }^{(1,2)}$. La necesidad crónica de suplementación intravenosa determina la falla intestinal crónica (FIC) o tipo III ${ }^{(1,2)}$. La nutrición parenteral (NP) es el tratamiento de sostén esencial para pacientes con FI y define su condición; si bien los pacientes sobreviven, las complicaciones asociadas con la NP crónica (NPC) son múltiples, pueden ser graves y frecuentemente condicionan la evolución. No obstante, la NPC sigue siendo el tratamiento estándar para los pacientes con FIC. La modalidad de NPC domiciliaria (NPD) como soporte vital está destinada estratégicamente a mantener el estado nutricional e hidroelectrolítico, así como mejorar la calidad de vida (CV), minimizando las complicaciones del uso a largo plazo de la NPC hospitalaria (infección relacionada con el catéter y trombosis venosa de los angioaccesos). Así como también reducir la probabilidad de enfermedad hepática (principales indicaciones del trasplante intestinal), enfermedad ósea metabólica, deterioro de la función renal y los costos asociados ${ }^{(3-5)}$. En Europa, se estima que la prevalencia de NPD para FIC oscila entre 5 y 80 por millón de habitantes, con una incidencia que varía entre 7,7 y 15 pacientes con FIC dependiente de NPC por millón de habitantes por año ${ }^{(6,7)}$. Lamentablemente, en la actualidad la NPD no forma parte de las prestaciones obligatorias en nuestro país, lo que determina una barrera de accesibilidad a esta técnica de soporte vital para un número cada vez mayor de pacientes que la necesitan. Resulta así completamente inaccesible para poblaciones de bajos recursos, existiendo únicamente casos puntuales en nuestro país, como el del paciente que presen$\operatorname{tamos}^{(7)}$. Por otro lado, el procedimiento quirúrgico más utilizado para convertir una anatomía altamente desfavorable en una más favorable para la rehabilitación del intestino es la cirugía de rehabilitación autóloga del tracto gastrointestinal (CRATGI), proceso que involucra a todos los procedimientos que tienen como objetivo reconstruir el tracto gastrointestinal, reclutar segmentos intestinales abandonados en el abdomen y cerrar ostomías, restableciendo la continuidad del tránsito intestinal ${ }^{(8-11)}$. Este procedimiento, cuando es desarrollado por un equipo experimentado, junto a otras estrategias de alargamiento intestinal, ha modificado la historia natural de la enfermedad. Permite aumentar el número de pacientes capaces de lograr la autonomía intestinal y la independencia de NPC. Los avances quirúrgi- cos, en cuidados intensivos y en NP, junto a la consolidación de unidades multidisciplinarias de terapia nutricional e insuficiencia intestinal para el tratamiento de la FIC en las últimas décadas, han permitido que dos tercios de los pacientes que desarrollan el SIC sobrevivan a su hospitalización inicial, y que un número similar sobreviva al primer año luego de desarrollar la FIC. Sin embargo, poco más de la mitad de los adultos con FIC logran independizarse completamente de la NPC dentro de los 5 años posteriores al diagnóstico y menos del 6\% abandonarán la NP utilizando métodos convencionales si no se logra la independencia en los primeros 2 años posteriores a la resección intestinal ${ }^{(12)}$. Afortunadamente, en los últimos años la atención de estos pacientes se centró en promover la rehabilitación intestinal utilizando un análogo recombinante del péptido 2 similar al glucagón humano (sGLP-2) producido mediante tecnología de ADN recombinante a partir de E. coli. El sGLP-2 (teduglutide) se ha convertido en la primera opción médica para pacientes seleccionados con FIC secundaria a SIC que no han logrado independencia a la NPD con la optimización del tratamiento estándar, con el potencial de reducir o eliminar la $\mathrm{NP}^{(13,14)}$. Presentamos el primer paciente en Uruguay con FIC que se trata con teduglutide luego de 17 años de dependencia a la NPC.

\section{Caso clínico}

Se trata de un paciente de sexo masculino de 24 años, estudiante universitario, que a los 6 años (31/8/2002) presentó un infarto intestino mesentérico extenso en el contexto de una panarteritis nodosa, requiriendo una enterectomia masiva. Su remanente intestinal presentaba una longitud intestinal postquirúrgica (LIPQ) de aproximadamente $10 \mathrm{~cm}(7 \mathrm{~cm}$ de yeyuno y $3 \mathrm{~cm}$ de íleon) con una anatomía postquirúrgica (APQ) tipo III (anastomosis yeyuno ileal con válvula ileocecal preservada y colon en continuidad) ${ }^{(18)}$. La asistencia del paciente que presentamos fue conducida por un equipo de referencia durante su edad pediátrica en Uruguay. Luego de la CRATGI en 2002, recibió NPC de forma ininterrumpida, completando 17 años de NPD. El paciente desarrolló múltiples complicaciones graves asociadas a la NPC y a la FIC, destacándose la enfermedad hepática (biopsia de julio de 2015: METAVIR estadio 2, fibrosis moderada). Por lo que fue contactado con la Unidad de Soporte Nutricional Clínico, Rehabilitación y Trasplante de Intestino del Hospital Universitario de la Fundación Favaloro en Argentina donde fue evaluado, dados los factores predictores negativos para rehabilitación con tratamiento estándar y la elevada probabilidad de requerir trasplante hepatointestinal. En la tabla 1 se observan los factores predictivos negativos que presenta- 
Tabla 1. Predictores negativos para la rehabilitación del intestino con tratamiento estándar.

\begin{tabular}{lll}
\hline & Factores predictivos negativos & Factores del paciente presentado \\
\hline Longitud intestinal postquirúrgica (LIPG) & Menor de $75 \mathrm{~cm}$ & Menos de $15 \mathrm{~cm}$ \\
Anatomía intestinal postquirúrgica (AIPQ) & Menos del $50 \%-60 \%$ del colon en continuidad (tipo I y II) & Todo el colon en continuidad (tipo III) \\
Tiempo de dependencia a la NPC & Mayor de 5 años & 18 años \\
Concentración de citrulina en sangre & Menor de $20 \mu \mathrm{mol} / \mathrm{L}$ & $13 \mu \mathrm{mol} / \mathrm{L}$ \\
Salud del intestino remanente & Intestino remanente enfermo & Intestino remanente sano \\
\hline
\end{tabular}

Tabla 2. Objetivos del tratamiento con sGLP-2.

\begin{tabular}{ll}
$\begin{array}{ll}\text { Número de deposiciones } \\
\text { Producción de orina: }\end{array}$ & Menos de cinco por día (Bristol 5-6) \\
Composición corporal & Mantenimiento del IMC \\
Capacidad funcional & $\begin{array}{l}\text { Mejoría del rendimiento físico, } \\
\text { dinamometría de pulso }\end{array}$ \\
Paraclínica & $\begin{array}{l}\text { Mantenimiento de los parámetros } \\
\text { normales (hematología, función renal y } \\
\text { hepática, electrolitos, vitaminas) }\end{array}$ \\
\hline
\end{tabular}

IMC: índice de masa corporal.

ba el paciente. Posteriormente el mismo fue referenciado a nuestro equipo para tratamiento y seguimiento conjunto de su FIC en la edad adulta. Desde 2017 recibe NPD nocturna en forma cíclica con emulsiones lipídicas de alternativa ahorradoras de lípidos omega 6. En ese mismo año se realizó nueva biopsia hepática que no evidenció cambios con respecto a la previa. Nuestro paciente inició el tratamiento con sGLP-2 (teduglutide) el 12 de diciembre de 2019, luego de un recurso de amparo en la justicia por uso compasivo, dado que es un medicamento de alto costo. Los objetivos para el manejo médico y nutricional con este fármaco se observan en la tabla 2. Se administró sGLP-2 $0,05 \mathrm{mg} / \mathrm{kg} /$ día una vez al día por vía subcutánea aproximadamente a la misma hora cada día y los controles se programaron a las 2, 4, $8,12,16,18,20$ y 24 semanas. Posteriormente, se programaron visitas de seguimiento cada 2 meses. Al inicio del tratamiento con sGLP-2, la NPD se administraba en infusión de 12 horas diarias $4-5$ veces a la semana por catéter tunelizado de larga duración. En la tabla 3 se muestra la estrategia de dosificación de teduglutide con relación a la función renal. El aporte nutricional semanal por vía parenteral y la evolución del resto de las variables estudiadas se observan en la tabla 4 . El paciente
Tabla 3. Estrategia de dosificación de teduglutide con relación a la función renal.

\begin{tabular}{|ll}
\hline Categoría & \multicolumn{1}{c}{ Dosis sugerida } \\
\hline Función renal normal & $\begin{array}{l}\text { Administrar una vez al día 0,05 } \\
\text { mg/kg mediante inyección } \\
\text { subcutánea }\end{array}$ \\
$\begin{array}{l}\text { Insuficiencia renal (aclaramiento } \\
\text { de creatinina inferior a } 50 \mathrm{ml} / \mathrm{min} \text { o } \\
\text { enfermedad renal en etapa }\end{array}$ \\
$\begin{array}{l}\text { terminal) } \\
\text { Modificada de Vu, Long. Chapter 14 - GLP-2 Agonists for Short Bo- }\end{array}$ \\
$\begin{array}{l}\text { wel Syndrome, Editor(s): Mandy L. Corrigan, Kristen Roberts, Ezra } \\
\text { Steiger. Adult Short Bowel Syndrome, Academic Press, 2019, Pages } \\
\text { 187-194, https://doi.org/10.1016/B978-0-12-814330-8.00014-7. }\end{array}$ \\
\end{tabular}

logró reducir el volumen semanal de NPD con respecto al que requería previo al inicio del tratamiento con sGLP-2 en un $25 \%$ en la octava semana de tratamiento. En la actualidad está completamente independizado de la NPD. Los objetivos del tratamiento se alcanzaron adecuadamente antes de la semana 24. Durante las primeras 76 semanas de seguimiento no ha presentado reacciones adversas ni complicaciones asociadas al tratamiento con sGLP-2.

\section{Discusión y comentarios}

Los pacientes con SIC desarrollan cuadros severos de desnutrición y deshidratación que comprometen la vida, además del normal desarrollo en los niños, de tal manera que requieren de una intervención nutricional especializada desde el inicio, de lo contrario no sobreviven. Si esta intervención tiene indicación de nutrición parenteral (NP), el cuadro se denomina falla intestinal $(\mathrm{FI})^{(1)}$. La FI ha sido la falla orgánica menos conocida y la última en ser reconocida hasta ahora. No fue hasta 2013 que la FIC debida a una enfermedad benigna se incluyó como un trastorno en la lista ORPHANET de en- 
Tabla 4. Respuesta al tratamiento con sGLP-2 (teduglutide) a la semana 12, 24 y 48 de seguimiento.

\begin{tabular}{|c|c|c|c|c|c|}
\hline Característica estudiada & Al iniciar sGLP-2 & $\begin{array}{l}\text { A la semana } 24 \text { de } \\
\text { seguimiento }\end{array}$ & $\begin{array}{c}\text { Variabilidad } \\
(0-\text { sem 24) (\%) }\end{array}$ & $\begin{array}{c}\text { A la semana } 48 \text { de } \\
\text { seguimiento }\end{array}$ & $\begin{array}{c}\text { Variabilidad } \\
(0-\text { sem 48) (\%) }\end{array}$ \\
\hline Peso (kg) & 57,2 & 57,8 & $1 \%$ & 60,6 & $5,8 \%$ \\
\hline IMC $\left(\mathrm{kg} / \mathrm{m}^{2}\right)$ & 18,68 & 18,87 & $1 \%$ & 19,76 & $5,8 \%$ \\
\hline NP (cal/sem) & 5.868 & 4.401 & $-25 \%$ & 600 & $-89,8 \%$ \\
\hline NP (L/sem) & 9,1 & 6,8 & $-25,3 \%$ & 1 & $-89 \%$ \\
\hline NP (días/sem) & 4 & 3 & $-25 \%$ & 1 & $-75 \%$ \\
\hline Diuresis (media en L/sem) & 2,5 & 3,7 & $48 \%$ & 2,7 & $8 \%$ \\
\hline \multicolumn{6}{|l|}{ Diuresis (L/día) } \\
\hline Días con NP & 1,2 & 3,8 & $216 \%$ & 3,5 & $192 \%$ \\
\hline Días sin NP & 0,9 & 3,7 & $311 \%$ & 2,1 & $189 \%$ \\
\hline \multicolumn{6}{|l|}{ Ingesta media de líquidos (L/día) } \\
\hline Días con NP & 3,1 & 3,5 & $13 \%$ & 3,0 & $-3 \%$ \\
\hline Días sin NP & 3,4 & 3,7 & $8,9 \%$ & 3,4 & $0 \%$ \\
\hline Deposiciones (veces por día) & 4 & 3 & -25 & 3 & -25 \\
\hline Deposiciones (escala de Bristol) & 7 & $6-7$ & $\mathrm{~N} / \mathrm{A}$ & $5-6$ & $N / A$ \\
\hline Ingesta oral (calorías/día) & 3.278 & 3.975 & 21,3 & 4.566 & $39,3 \%$ \\
\hline Creatininemia (mg/dL) & 0,84 & 0,75 & $\mathrm{~N} / \mathrm{A}$ & 0,81 & $\mathrm{~N} / \mathrm{A}$ \\
\hline Bilirrubina total (mg/dL) & 3,34 & 2,02 & $\mathrm{~N} / \mathrm{A}$ & 1,81 & $\mathrm{~N} / \mathrm{A}$ \\
\hline Plaquetas (× 109/L) & 170 & 151 & $\mathrm{~N} / \mathrm{A}$ & 179 & $\mathrm{~N} / \mathrm{A}$ \\
\hline $\begin{array}{l}\text { Dosificación de vitamina B } 12 \\
\text { cianocobalamina }(\mathrm{pg} / \mathrm{mL})\end{array}$ & 446 & $\mathrm{~N} / \mathrm{C}$ & $\mathrm{N} / \mathrm{A}$ & 452 & $N / A$ \\
\hline Citrulinemia $(\mu \mathrm{mol} / \mathrm{mL})$ & 13 & 10 & $\mathrm{~N} / \mathrm{A}$ & $\mathrm{N} / \mathrm{C}$ & $N / A$ \\
\hline Actividad física (h/sem) & 2 & 4 & $100 \%$ & 7 & $250 \%$ \\
\hline $\begin{array}{l}\text { Dinamometría de pulso }(\mathrm{kg}) \text {. } \\
\text { Mano dominante }\end{array}$ & 22 & 24 & $9 \%$ & 36 & $63,6 \%$ \\
\hline
\end{tabular}

N/A: no aplica; N/C: no contamos.

fermedades raras (Código ORPHA: 294422) ${ }^{(15)}$. La posibilidad de reversibilidad de la falla intestinal crónica depende de múltiples factores que se observan en la tabla $1^{(16)}$. Los pacientes incapaces de rehabilitación se mantienen en NPD como única alternativa, con una sobrevida a los 5 años cercana al $80 \%{ }^{(17,18)}$.

Actualmente la NP continúa siendo la principal terapéutica para pacientes con FI, sin embargo su uso a largo plazo puede generar complicaciones que determinen la necesidad de trasplante de intestino, combinado (hígado-intestino) o multivisceral. ${ }^{(3-7,18-20)}$ No obstante, a pe- sar del desarrollo del trasplante de intestino a nivel mundial, los resultados no han sido los deseados. A esto se suma que existen importantes limitaciones de accesibilidad al procedimiento ${ }^{(15-21)}$. Estos hechos han reflotado el interés en el campo de la rehabilitación de pacientes con FIC. Se ha promovido la rehabilitación intestinal como objetivo primario para recuperar la suficiencia del intestino e independizarse de la NPC. Los avances en las técnicas quirúrgicas y sobre todo en el tratamiento médico han hecho posible que, hasta pacientes con menos de $20 \mathrm{~cm}$ de intestino puedan alimentarse e hidratarse por 


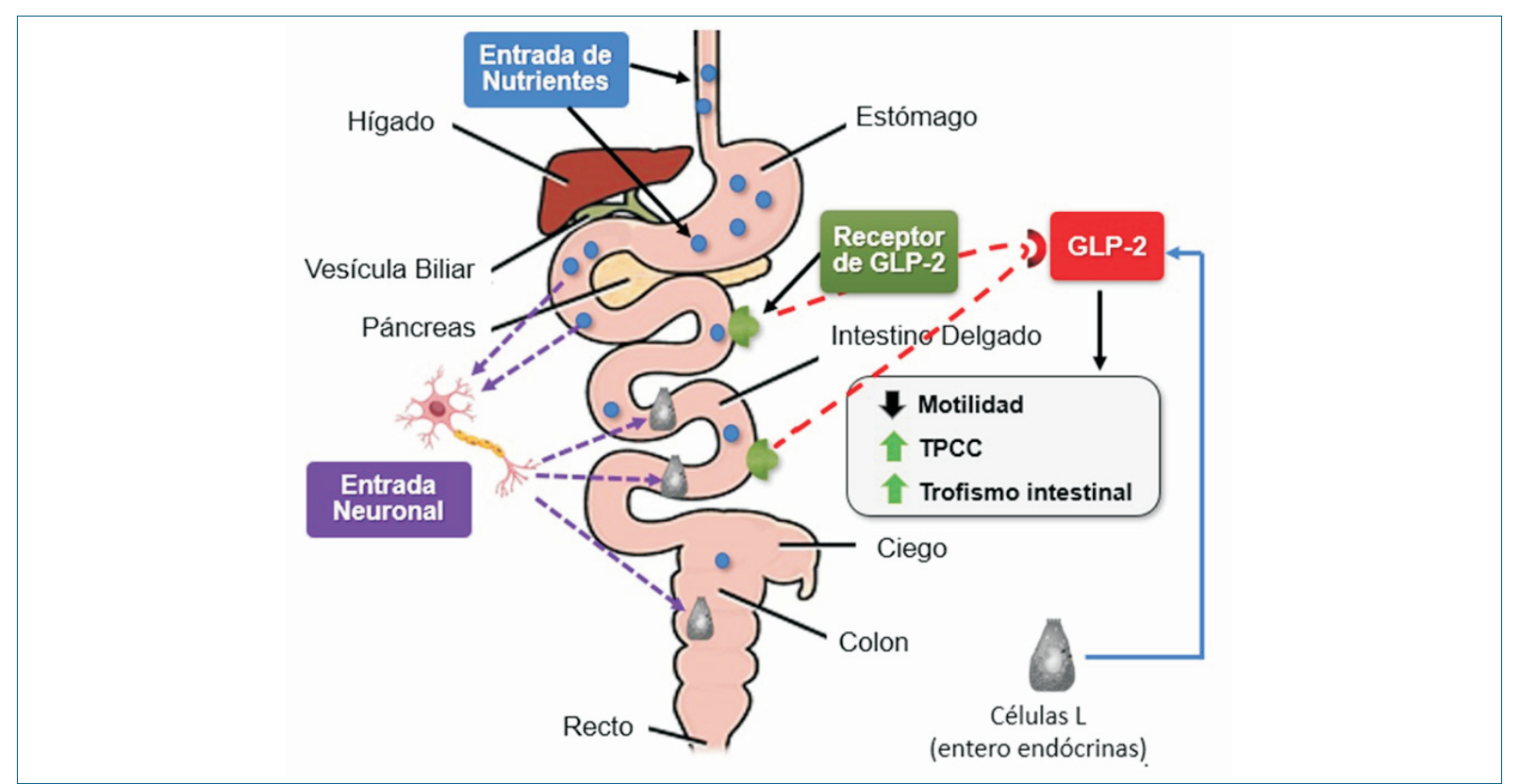

Figura 1A. Esquema del mecanismo de acción del GLP-2 a nivel intestinal.

El GLP-2 (péptido 2 similar al glucagón) es liberado por las células L del íleon distal y el colon y actúa de forma aguda para enlentecer la motilidad proximal y aumenta de forma crónica el área de superficie mucosa, aumentando la capacidad de transporte de nutrientes. TPCC: tasa de producción de células de cripta. Modificado de: Sigalet DL. Nonruminant Nutrition Symposium: The role of glucagon-like peptide-2 in controlling intestinal function in human infants: regulator or bystander? J Anim Sci. 2012 Apr;90(4):1224-32. doi: 10.2527/jas.2011-4704.

vía oral sin necesidad de NP o trasplante ${ }^{(15-21)}$. Los pacientes con FIC que han logrado resultados favorables en cuanto a su sobrevida y rehabilitación son particularmente aquellos que accedieron a una NPD segura y de calidad, que fueron tratados en centros especializados que cuentan con un programa multidisciplinario compuesto por clínicos, cirujanos, gastroenterólogos, nutriólogos, nutricionistas, enfermeros, psicólogos, intensivistas, infectólogos, paliativistas y asistentes sociales. Estos equipos logran una gestión adecuada de la catástrofe abdominal que genera el problema y de la falla intestinal secundaria. En conjunto tienen como principales objetivos devolverles la suficiencia intestinal reduciendo o eliminando la dependencia a la NPC y mejorando su CV. Al mismo tiempo que optimizan los costos relacionados con la asistencia de estos pacientes ${ }^{(22)}$. Uno de los resultados más relevantes publicados hasta la fecha es que el manejo médico y nutricional, realizado por un equipo de referencia, permite reducir significativamente los requerimientos de NPD (en hasta 40\%) en la mayoría de los pacientes antes de considerar el uso de sGLP-2 ${ }^{(23,24)}$. Por lo que el esfuerzo debe destinarse a la conformación de estos equipos y este fármaco nunca deba ser considerado como el primer sustituto de la NPD. La tasa de mortalidad de los pacientes con FIC con NPD es de aproximadamente $20 \%-30 \%$ después de 5 años, que es aproximadamente igual a la supervivencia de los pacientes después del trasplante intestinal ${ }^{(25)}$. En 2012 el sGLP-2 fue aprobado por la FDA. La probabilidad de alcanzar la suficiencia intestinal, incluso en pacientes con anatomía desfavorable, ha aumentado con su introducción en los protocolos de tratamiento para pacientes seleccionados ${ }^{(8-10,13,26)}$. Actualmente en centros de referencia, únicamente aquellos pacientes que no pudieron continuar reduciendo el volumen de NPD en un período de aproximadamente 6 meses, son evaluados para iniciar sGLP-2. Existen fórmulas predictivas disponibles para estimar la probabilidad de destete de la NPD en este grupo de pacientes, lo que facilita una evaluación objetiva que evita indicaciones inadecuadas de un medicamento de alto costo ${ }^{(11,12,27)}$.

Las células L intestinales enteroendocrinas producen proglucagón que se escinde para formar glucagón, así como múltiples fragmentos de péptido derivado de proglucagón (PGDP), incluidos el péptido 1 similar al glucagón (GLP-1) y el péptido 2 similar al glucagón (GLP-2), denominados así porque comparten aproximadamente el 50\% de los mismos aminoácidos que el glucagón ${ }^{(28)}$. Estos PGDP y el glucagón comparten acciones relacionadas que regulan el equilibrio de nutrientes 


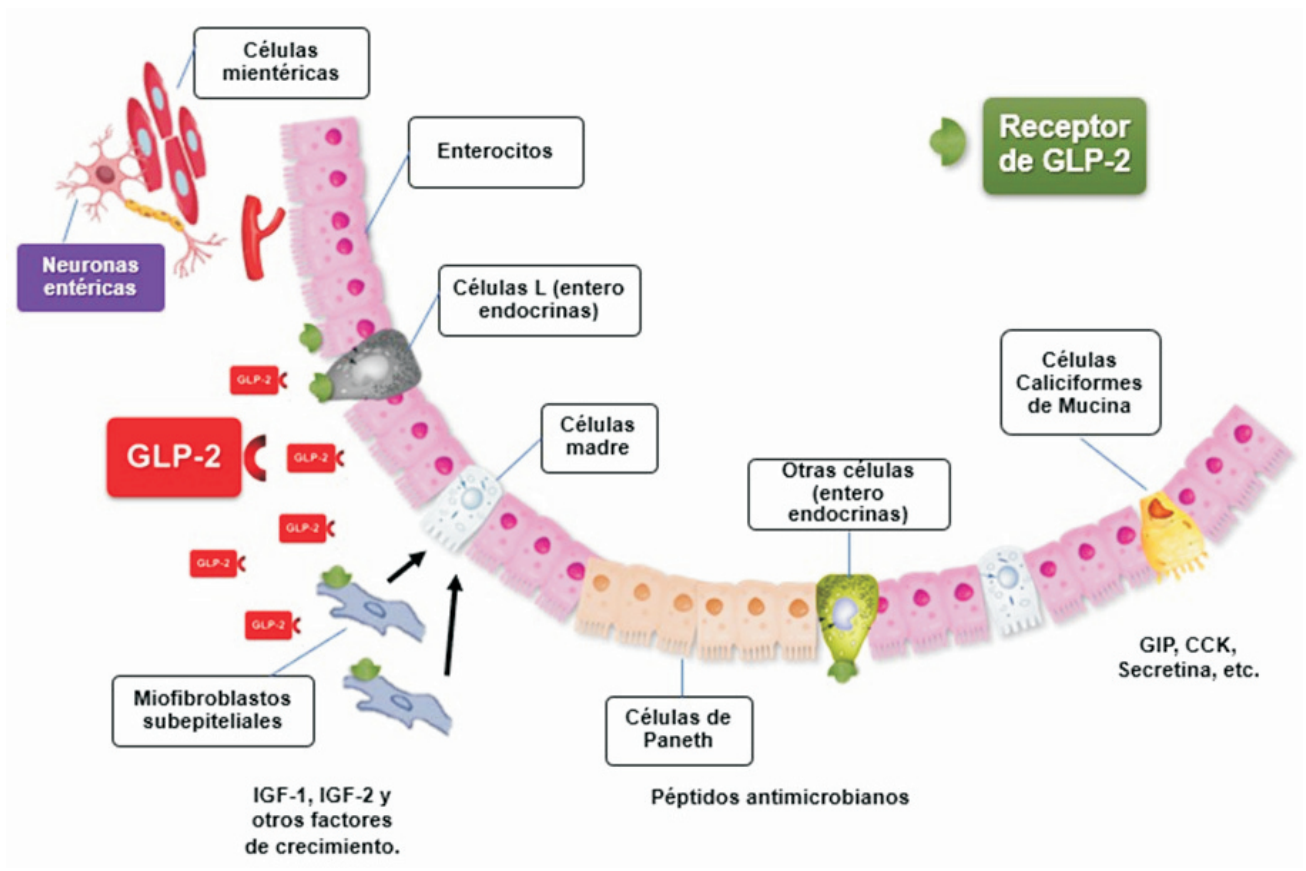

Figura 1B. Efectos del péptido 2 similar al glucagón y sus mediadores sobre las células diana en el tracto gastrointestinal.

La activación de los receptores de GLP-2 (GLP-2R) en las neuronas entéricas estimula la liberación de óxido nítrico (ON) y péptido intestinal vasoactivo (VIP). El ON aumenta el flujo sanguíneo mesentérico y aumenta la captación de aminoácidos y glucosa, y el VIP reduce la infiltración de células inmunes, la liberación de citocinas proinflamatorias y la apoptosis en los enterocitos. Los factores de crecimiento liberados de los miofibroblastos subepiteliales por activación de GLP-2R, incluido el factor de crecimiento similar a la insulina (IGF) -1, IGF-2, el factor de crecimiento de queratinocitos (KGF) y el factor de crecimiento epidérmico (EGF)-miembros de la familia ErbB, median el aumento de la proliferación y crecimiento de diversas células de las criptas intestinales. La expresión de las proteínas de unión estrecha celular que controlan la permeabilidad intestinal se ve afectada por el GLP-2 y depende del receptor de IGF-1. La síntesis y la actividad normales de los péptidos antimicrobianos producidos por las células de Paneth dependen de la activación de GLP-2R. Otras células enteroendocrinas que secretan factores como el péptido inhibidor gástrico (GIP), la secretina y la colecistoquinina (CCK) expresan GLP-2R y tienen varios efectos posteriores.

Modificado de: Connor EE, Evock-Clover CM, Wall EH, Baldwin RL 6th, Santin-Duran M, Elsasser TH, Bravo DM. Glucagon-like peptide 2 and its beneficial effects on gut function and health in production animals. Domest Anim Endocrinol. 2016 Jul;56 Suppl:S56-65. doi: 10.1016/j.domaniend.2015.11.008.

y energía. La unión de GLP-2 a su receptor en las células intestinales determina a una mayor absorción de carbohidratos, aminoácidos y lípidos directamente a través del crecimiento de las células epiteliales intestinales, lo que provoca un aumento en el área de superficie de la mucosa de absorción (aumento de la altura de las vellosidades del intestino delgado, la profundidad de las criptas, el peso intestinal ${ }^{(29)}$. Los estudios en animales y humanos muestran un aumento del flujo sanguíneo visceral y portal con la infusión de GLP- $2^{(30)}$. También puede inhibir la motilidad gastrointestinal y la secreción de ácido gástrico para aumentar el tiempo de absorción de nutrientes ${ }^{(31)}$. Estas acciones intestinotróficas y antia- poptóticas se consideraron el objetivo terapéutico para el tratamiento del SIC y la FIC (figura 1). Estos cambios estructurales y funcionales a nivel intestinal son los que han permitido reducir o eliminar la dependencia a la NP en hasta $60 \%$ de los pacientes con una media de 50 semanas de tratamiento, así como ocurrió con el caso que presentamos $^{(31-36)}$. Los pacientes se consideran "respondedores tempranos" si el volumen de NP se reduce más del 20\% del valor inicial antes de la semana 24 , y "respondedores tardíos" si la respuesta positiva ocurre después de 24 semanas de tratamiento con sGLP- $2^{(27)}$. Nuestro paciente logró reducir un $25 \%$ del volumen de NPD a la semana 8 , sin presentar efectos adversos signi- 


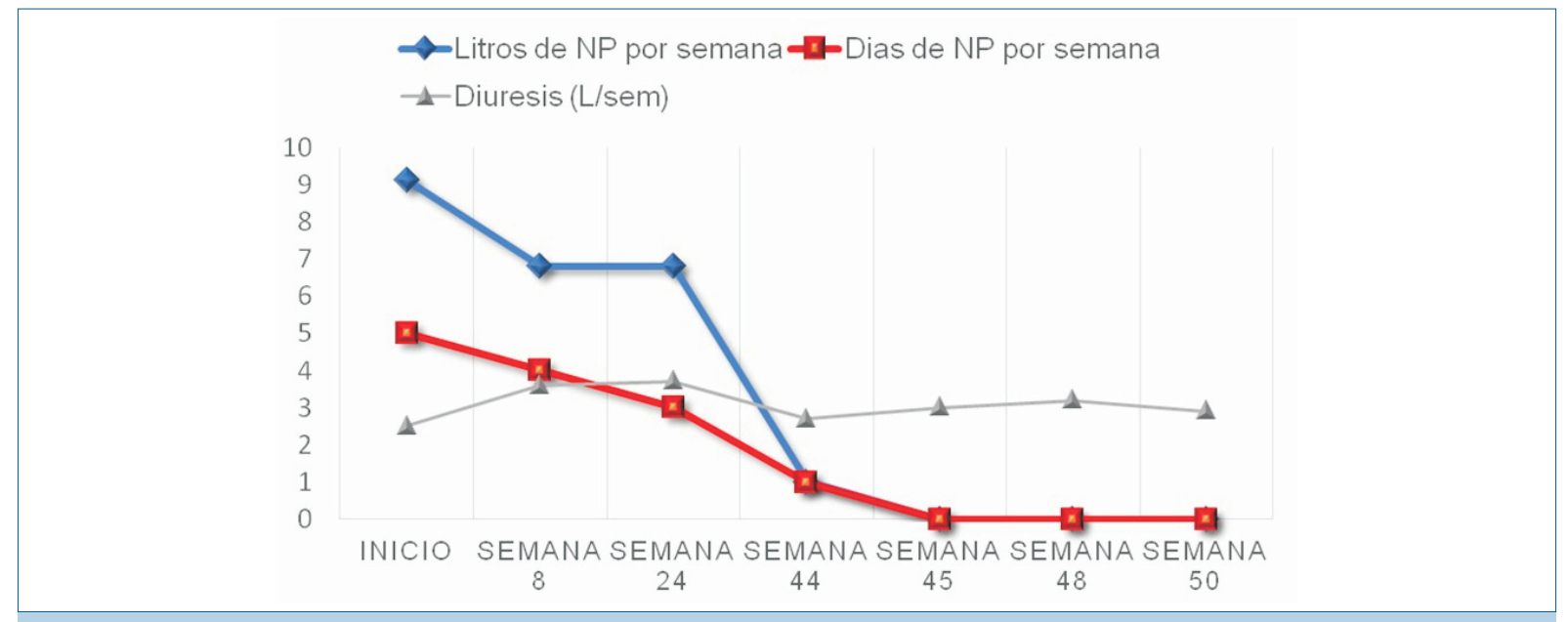

Figura 2. Evolución de los requerimientos de nutrición parenteral domiciliaria y la media de diuresis semanal desde el inicio del tratamiento con sGLP-2 (Teduglutide). NP: nutrición parenteral.

ficativos que obligaran a suspender el tratamiento. En la figura 2 se observa la evolución de los requerimientos de NPD en relación con el tiempo de tratamiento. Las demás variables de resultado se ven en la tabla 4. Al mismo tiempo se observó una mejoría en su composición corporal, así como su capacidad funcional evaluada por dinamometría de pulso. Las perspectivas a futuro con este paciente son lograr la independencia completa de la NPD y suspender posteriormente el sGLP-2. Actualmente (semana 76 de tratamiento) el paciente cursa su semana 30 sin necesitar NP. Según resultados alentadores recientemente publicados, aproximadamente la mitad de los pacientes que ya no recibían NPD pudieron interrumpir el tratamiento con sGLP-2, con un tiempo medio de tratamiento posterior de 63,5 semanas (DE \pm 45,2). Manteniendo el peso, estado funcional, diuresis, número de deposiciones y estabilidad del medio interno, probablemente vinculado a su efecto acumulativo ${ }^{(27)}$.

\section{Conclusiones}

La incidencia de pacientes con SIC y FIC ha aumentado a lo largo de los años. El progreso de la medicina de cuidados intensivos y las técnicas de nutrición parenteral han mejorado significativamente el pronóstico de individuos que han perdido gran parte de su tracto intestinal como nuestro paciente. Presentamos la versión narrativa de un caso clínico único en nuestro país, un paciente con FIC y factores predictivos clásicos negativos para la rehabilitación intestinal con tratamiento estándar. La NPD segura y de alta calidad, conducida por un equipo especializado, ha permitido la supervivencia de este paciente, que sin embargo no estuvo exenta de complicaciones a largo plazo. El sGLP-2 ha revolucionado el tratamiento de la FIC y permitió la independencia de la
NPD como ocurre en un porcentaje significativo de pacientes. Se impone la necesidad de establecer y consolidar equipos multidisciplinarios con experiencia en el manejo de los pacientes con falla intestinal en nuestro país.

\section{Abstract}

Long-term home parenteral nutrition (HPN) is a life-saving treatment for patients with chronic intestinal failure, an invalidating condition. However, only 1 out of 3 countries can rely on this treatment and complications associated to chronic parenteral nutrition are rather frequent. The latter constitute the main indication for intestinal transplantion, a procedure that in most middle-income countries has not yet developed and has not shown the expected outcome. In recent years, intestinal rehabilitation has significantly improved at the global level with the use of GLP2, based on the growing evidence that proves the possibility of intestinal rehabilitation and independence from parenteral nutrition with Teduglutide, even in the case of patients with unfavorable anatomy. These results have caused a positive impact on survival and the quality of life of patients with chronic renal failure, and they can often abstain from transplant.

The patient of the case study is the first one who received this therapy in our country and this article analyses his favorable early response to treatment and future perspectives.

\section{Resumo}

A insuficiência intestinal crônica (CIF) ou tipo III é uma condição incapacitante e a nutrição parenteral crônica (NPC) domiciliar é o tratamento que permite a 
sobrevida desses pacientes. No entanto, apenas $1 \mathrm{em}$ cada 3 países latino-americanos dispõe desse recurso e as complicações da NPC não são raras. Essas complicações são as principais indicações para o transplante intestinal, procedimento que na maioria dos países de renda média não foi desenvolvido ou não apresentou os resultados esperados. Nos últimos anos, a reabilitação intestinal em todo o mundo tem melhorado substancialmente com o uso de sGLP2, com um número cada vez maior de evidências que mostram a possibilidade de reabilitação intestinal e independência da NPC, mesmo em pacientes com anatomia desfavorável. Esses resultados têm possibilitado prolongar a sobrevida e melhorar a qualidade de vida dos pacientes com CIF e, em muitos casos, dispensar o transplante. $O$ paciente do caso que apresentamos é o primeiro a receber essa terapia em nosso país. Neste artigo, analisamos a resposta favorável ao tratamento precoce e suas perspectivas futuras.

\section{Bibliografía}

1. Pironi L. Definitions of intestinal failure and the short bowel syndrome. Best Pract Res Clin Gastroenterol 2016; 30(2):173-85.

2. Pironi L. Revised ESPEN Clinical Classification Of Chronic Intestinal Failure: from 16 to 8 categories. JPEN J Parenter Enteral Nutr 2017; 41(6):911.

3. Pironi L, Goulet O, Buchman A, Messing B, Gabe S, Candusso $\mathrm{M}$, et al. Outcome on home parenteral nutrition for benign intestinal failure: a review of the literature and benchmarking with the European prospective survey of ESPEN. Clin Nutr 2012; 31(6):831-45.

4. Lal S, Pironi L, Wanten G, Arends J, Bozzetti F, Cuerda C, et al. Clinical approach to the management of Intestinal Failure Associated Liver Disease (IFALD) in adults: a position paper from the Home Artificial Nutrition and Chronic Intestinal Failure Special Interest Group of ESPEN. Clin Nutrit 2018; 37(6 Pt A):1794-7.

5. Joly F, Baxter J, Staun M, Kelly DG, Hwa YL, Corcos O, et al. Five-year survival and causes of death in patients on home parenteral nutrition for severe chronic and benign intestinal failure. Clinical Nutr 2018; 37(4):1415-22.

6. Pironi L, Arends J, Bozzetti F, Cuerda C, Gillanders L, Jeppesen PB, et al. ESPEN guidelines on chronic intestinal failure in adults. Clin Nutr 2016; 35(2):247-307.

7. Moreira E, López P, Silva L, Olano E. Dependiendo de la nutrición parenteral para sobrevivir. Falla intestinal crónica por síndrome de intestino corto. Comunicación de un caso y revisión de la literatura Rev Méd Urug 2019; 35(4):316-24. doi: 10.29193/RMU.35.4.8.

8. Sudan D, Rege A. Update on surgical therapies for intestinal failure. Curr Opin Organ Transplant 2014; 19(3):267-75.

9. Gondolesi GE, Pattín F, Nikkoupur H. Management of intestinal failure in middle-income countries, for children and adults. Curr Opin Organ Transplant 2018; 23(2):212-8.
10. Gondolesi GE, Doeyo M, Echevarria C, Lobos F, Rubio S, Rumbo C, et al. Results of surgical and medical rehabilitation for adult patients with type III intestinal failure in a comprehensive unit today: building a new model to predict parenteral nutrition independency. JPEN J Parenter Enteral Nutr 2020; 44(4):703-13.

11. Abu-Elmagd KM, Armanyous SR, Fujiki M, Parekh NR, Osman M, Scalish M, et al. Management of five hundred patients with gut failure at a single center: surgical innovation versus transplantation with a novel predictive model. Ann Surg 2019; 270(4):656-74.

12. Kelly DG, Tappenden KA, Winkler MF. Short bowel syndrome: highlights of patients management, quality of life, and survival. JPEN J Parenter Enteral Nutr 2014; 38(4):427-37.

13. Schwartz LK, O'Keefe SJD, Fujioka K, Gabe SM, Lamprecht G, Pape UF, et al. Long-term teduglutide for the treatment of patients with intestinal failure associated with short bowel syndrome. Clin Transl Gastroenterol 2016; 7(2):e142.

14. Seidner DL, Fujioka K, Boullata JI, Iyer K, Lee HM, Ziegler TR. Reduction of parenteral nutrition and hydration support and safety with long term teduglutide treatment in patients with short bowel syndrome-associated intestnal failure: STEPS-3 study. Nutr Clin Pract 2018; 33(4):520-7.

15. Orphanet. El portal sobre enfermedades raras y medicamentos huérfanos. Disponible en: http://www.orpha.net (Consulta: 2 noviembre 2020).

16. Amiot A, Messing B, Corcos O, Panis Y, Joly F. Determinants of home parenteral nutrition dependence and survival of 268 patients with non-malignant short bowel syndrome. Clin Nutr 2013; 32(3):368-74.

17. Noelting J, Gramlich L, Whittaker S, Armstrong D, Marliss E, Jurewitsch B, et al. Survival of patients with short-bowel syndrome on home parenteral nutrition: a prospective cohort study. JPEN J Parenter Enteral Nutr 2020. doi: 10.1002/jpen.1984.

18. Fishbein TM, Gondolesi GE, Kaufman SS. Intestinal transplantation for gut failure. Gastroenterology 2003; 124(6):1615-28.

19. Abu-Elmagd K. The concept of gut rehabilitation and the future of visceral transplantation. Nat Rev Gastroenterol Hepatol 2015; 12(2):108-20.

20. Kaufman SS, Avitzur Y, Beath SV, Ceulemans LJ, Gondolesi GE, Mazariegos GV, et al. New insights into the indications for intestinal transplantation: consensus in the year 2019. Transplantation 2019; 104(5):937-46.

21. Gondolesi GE, Doeyo M, Solar-Muñiz H. A detailed analysis of the current status of intestinal transplantation in Latin America. Curr Opin Organ Transplant 2020; 25(2):189-95.

22. Amiot A, Messing B, Corcos O, Panis Y, Joly F. Determinants of home parenteral nutrition dependence and survival of 268 patients with non-malignant short bowel syndrome. Clin Nutr 2013; 32(3):368-74. 
23. Noelting J, Gramlich L, Whittaker S, Armstrong D, Marliss E, Jurewitsch B, et al. Survival of patients with short-bowel syndrome on home parenteral nutrition: a prospective cohort study. JPEN J Parenter Enteral Nutr 2020. doi: 10.1002/jpen.1984.

24. Lam K, Schwartz L, Batisti J, Iyer KR. Single-center experience with the use of teduglutide in adult patients with short bowel syndrome. JPEN J Parenter Enteral Nutr 2018; 42(1):225-30.

25. Schalamon J, Mayr JM, Höllwarth ME. Mortality and economics in short bowel syndrome. Best Pract Res Clin Gastroenterol 2003; 17(6):931-42. doi: 10.1016/s1521-6918(03)00079-9.

26. Jeppesen PB, Gilroy R, Pertkiewicz M, Allard JP, Messing B, O'Keefe SJ. Randomised placebo-controlled trial of teduglutide in reducing parenteral nutrition and/or intravenous fluid requirements in patients with short bowel syndrome. Gut 2011; 60(7):902-14.

27. Solar H, Doeyo M, Ortega M, De Barrio S, Olano E, Moreira $\mathbf{E}$, et al. Postsurgical intestinal rehabilitation using semisynthetic glucagon ] like peptide ] 2 analogue (sGLP ] 2) at a referral center: ¿Can patients achieve parenteral nutrition and sGLP ] 2 independency? JPEN J Parenter Enteral Nutr 2020. doi:10.1002/jpen.1983.

28. Drucker DJ, Yusta B. Physiology and pharmacology of the enteroendocrine hormone glucagon-like peptide-2. Annu Rev Physiol 2014; 76:561-83. doi: 10.1146/annurev-physiol-021113-170317.

29. Tappenden KA, Edelman J, Joelsson B. Teduglutide enhances structural adaptation of the small intestinal mucosa in patients with short bowel syndrome. J Clin Gastroenterol 2013; 47(7): 602-7. doi: 10.1097/MCG.0b013e3182828f57.
30. Bremholm L, Hornum M, Andersen UB, Hartmann B, Holst JJ, Jeppesen PB. The effect of glucagon-like peptide-2 on mesenteric blood flow and cardiac parameters in end-jejunostomy short bowel patients. Regul Pept 2011; 168(1-3): 32-8. doi: 10.1016/j.regpep.2011.03.003.

31. O'Keefe SJ, Jeppesen PB, Gilroy R, Pertkiewicz M, Allard JP, Messing B. Safety and efficacy on teduglutide after 52 weeks of treatment in patients with short bowel intestinal failure. Clin Gastroenterol Hepatol 2013; 11(7):815-23.e1-3.

32. Iyer KR, Kunecki M, Boullata JI, Fujioka K, Joly F, Gabe $\mathbf{S}$, et al. Independence from parenteral nutrition and intravenous fluid support during treatment with teduglutide among patients with intestinal failure associated with short bowel syndrome. JPEN J Parenter Enteral Nutr 2017; 41(6):946-51.

33. Lam K, Schwartz L, Batisti J, Iyer KR. Single-center experience with the use of teduglutide in adult patients with short bowel syndrome. JPEN J Parenter Enteral Nutr 2018; 42(1):225-30.

34. Jeppesen PB, Gabe SM,Seidner DL, Lee HM, Olivier C. Factors associated with response to teduglutide in patients with short bowel syndrome and intestinal failure. Gastroenterology 2018; 154(4):874-85.

35. Compher C, Gilroy R, Pertkiewicz M, Ziegler TR, Ratcliffe SJ, Joly F, et al. Maintenance of parenteral nutrition volume reduction, without weight loss, after stopping teduglutide in a subset of patients with short bowel syndrome. JPEN J Parent Enteral Nutr 2011; 35(5):603-9.

36. Pevny S, Maasberg S, Rieger A, Karber M, Blüthner E, Knappe-Drzikova B, et al. Experience with teduglutide treatment for short bowel syndrome in clinical practice. Clin Nutr 2019; 38(4):1745-55.
Contribución de autores

Los autores participaron en igual medida en las distintas etapas de elaboración del artículo.

Eduardo Moreira, ORCID 0000-0002-2522-3230.

Lourdes Silva, ORCID 0000-0002-2259-2313.

Estela Olano, ORCID 0000-0002-8101-8979.

Héctor Solar, ORCID 0000-0001-8631-7073. 